\title{
Stachyurus praecox - Japanischer Perlschweif, eine attraktive Alternative zu Forsythien
}

\author{
Veit Martin Dörken \& Annette Höggemeier
}

\begin{abstract}
Stachyurus praecox is a medium sized Japanese shrub. It is an interesting plant for solitary arrangements in gardens and parks with an impressive flowering in early spring. As an elegant alternative to Forsythia species it deserves closer attention.
\end{abstract}

\section{Zusammenfassung}

Stachyurus praecox ist eine japanische Strauchart, die sich gut als Solitär in Gärten und Parkanlagen eignet. Im zeitigen Frühjahr beeindruckt sie durch einen reichen, hellgelben Blütenflor. Die Art ist eine elegante Alternative zu den allgegenwärtigen Forsythien und verdient mehr Beachtung bei der Pflanzenwahl für die Gestaltung von Parks und Gärten.
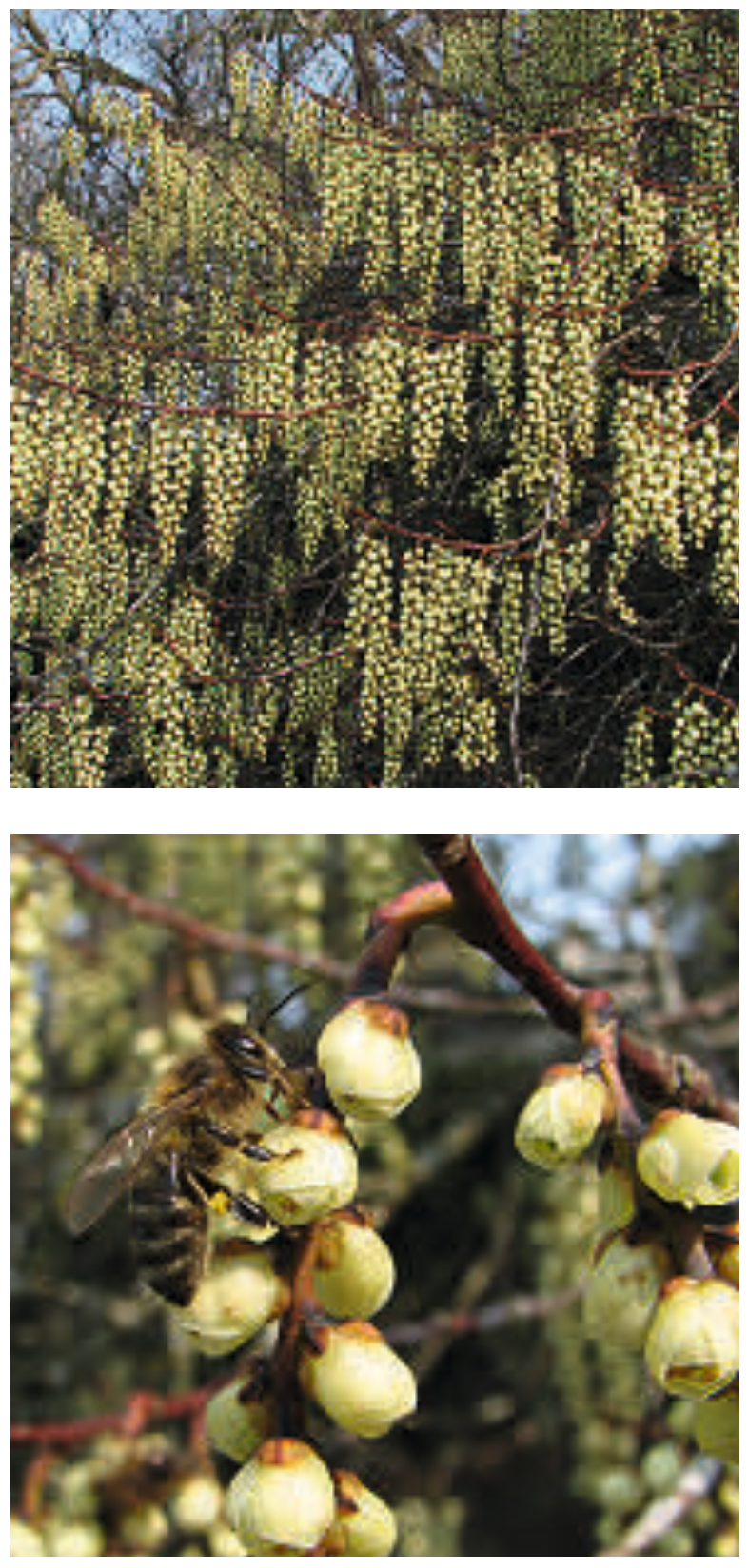

\section{Einleitung}

Lange Zeit war die Kornelkirsche (Cornus mas, Cornaceae) der einzige Vorfrühlingsblüher, der gelbe Farbtöne in unsere Gärten brachte. Seit Forsythien (darunter besonders die HybridForsythie, Forsythia x intermedia) mit ihrem überreichen Blütenflor Einzug in die mitteleuropäischen Baumschulsortimente gehalten hat, wurden sie in kürzester Zeit das dominierende gelb blühende Element in unseren Gärten und Parkanlagen. Dies liegt nicht zuletzt an ihrer Anspruchslosigkeit und Schnittverträglichkeit. Wenn hier die Lebensweisheit „varietas delectat" gilt, dann haben die Perlschweife (Stachyurus spp.) mehr Beachtung verdient.

\section{Systematik und Verbreitung}

Stachyurus ist die einzige Gattung innerhalb der Stachyuraceae. Sie umfasst fünf (oder sechs) Arten aus Japan und China (MabBerley 2008). In Mitteleuropa sind lediglich der Chinesische (S. chinensis) und der Japanische Perlschweif (S. praecox) winterhart. Der Japanische Perlschweif ist in Bergwäldern Japans, mit Ausnahme der nördlichsten Insel Hokkaido mit ihren langen und kalten Wintern, heimisch. Er gedeiht auf gleichmäßig feuchten, locker hu-

Abb. 1 (oben): Stachyurus praecox zur Blütezeit im März.

Abb. 2 (unten): Blüten mit Bienenbesuch. 
mosen Böden in schattigen bis halbschattigen Lagen, hat also vergleichbare Ansprüche wie Rhododendron.

\section{Namensgebung}

Die Familien- und Gattungsbezeichnung leitet sich aus dem Griechischen von stachys = Ähre und oura $=$ Schweif, Schwanz ab. Der Artbeiname praecox stammt aus dem Lateinischen und bedeutet so viel wie vorzeitig, was sich auf die Blütezeit im Vorfrühling bezieht.

\section{Morphologie}

Der Japanische Perlschweif ist ein zierlicher, weit ausladender winterkahler Strauch, der unter mitteleuropäischen Klimabedingungen etwa 2-3 $\mathrm{m}$ hoch und ähnlich breit wird. Seine rotbraunen Triebe sind weit ausladend und hängen bogig über. Die wechselständig angeordneten, bis $15 \mathrm{~cm}$ langen, elliptischen Blätter sind stark gezähnt und weisen eine deutlich kürzere Blattspitze als diejenigen von $S$. chinensis auf. Bereits im Vorjahr werden an den Trieben die Blütenstände angelegt, wobei die Blüten ohne besonderen Knospenschutz überwintern. Die hellgelben, glockenförmigen, kurz gestielten Blüten sind in bis $10 \mathrm{~cm}$ langen, straff herabhängenden, vielblütigen Trauben angeordnet. Die radiärsymmetrischen Einzelblüten sind vierzählig. Sie umfassen vier bräunlich-grüne Kelch- und vier hell- bis schwefelgelbe Kronblätter sowie zwei Kreise aus je vier Staubblättern. Der oberständige Fruchtknoten besteht aus vier verwachsenen Fruchtblättern. Der Griffel überragt die Kronblätter nicht und liegt in der glockigen Krone verborgen. Die Narbe ist vierzählig und kopfig. Die Blüten entfalten sich noch vor oder mit dem Laubaustrieb von März bis April über einen recht langen Zeitraum von drei bis fünf Wochen. Stachyurus praecox ist somit im Frühling eine wichtige Bienen- und Hummelweide (KrüSSMANN I978, Roloff \& BärTels 1996).

Abb. 3 (oben): Früchte.

Abb. 4 (Mitte): Blätter.

Abb. 5 (unten): Die Sorte 'Rubrifolius' mit rotem Herbstlaub im Steingarten des Palmengartens.
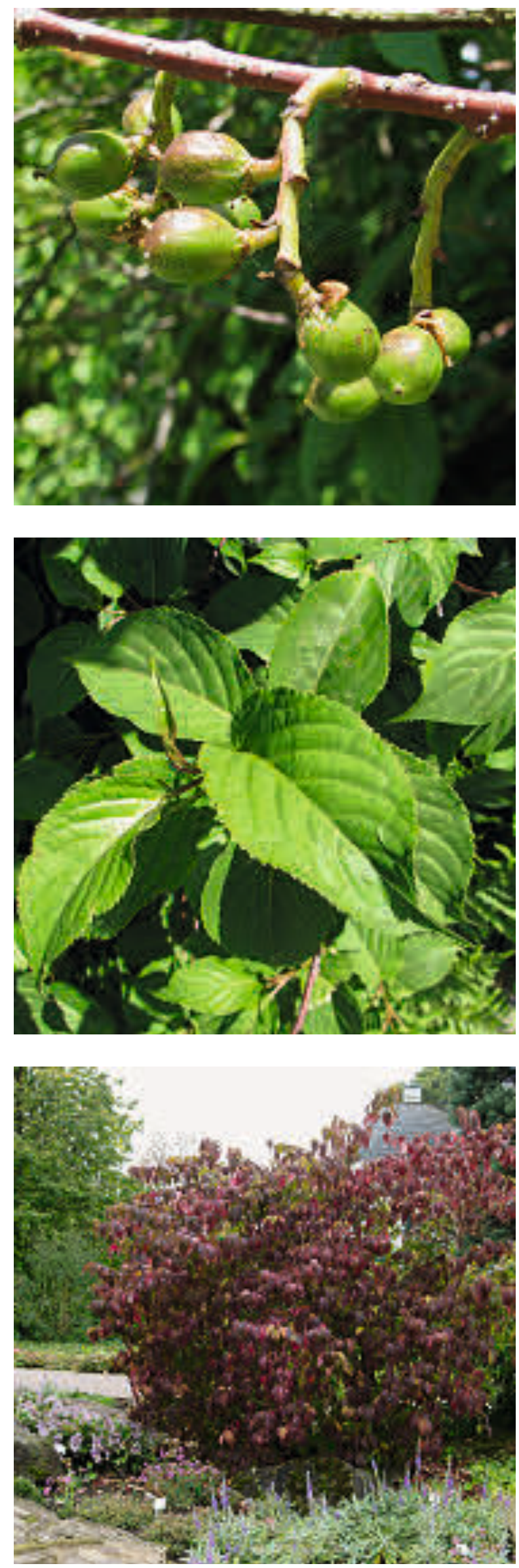

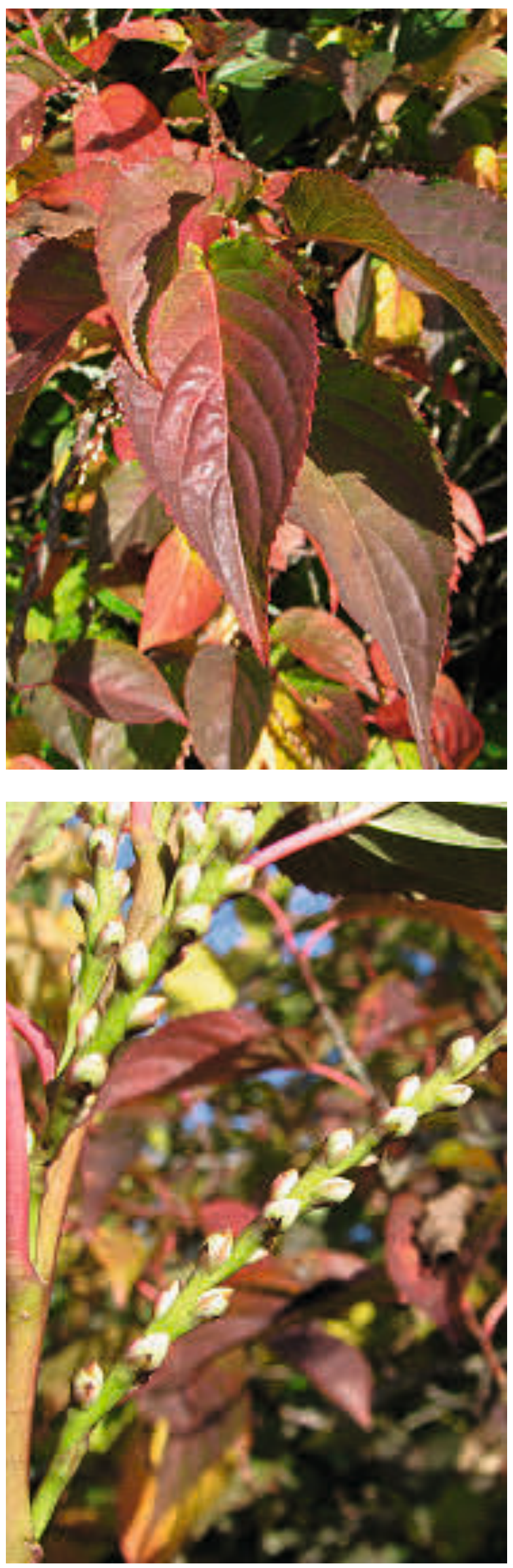

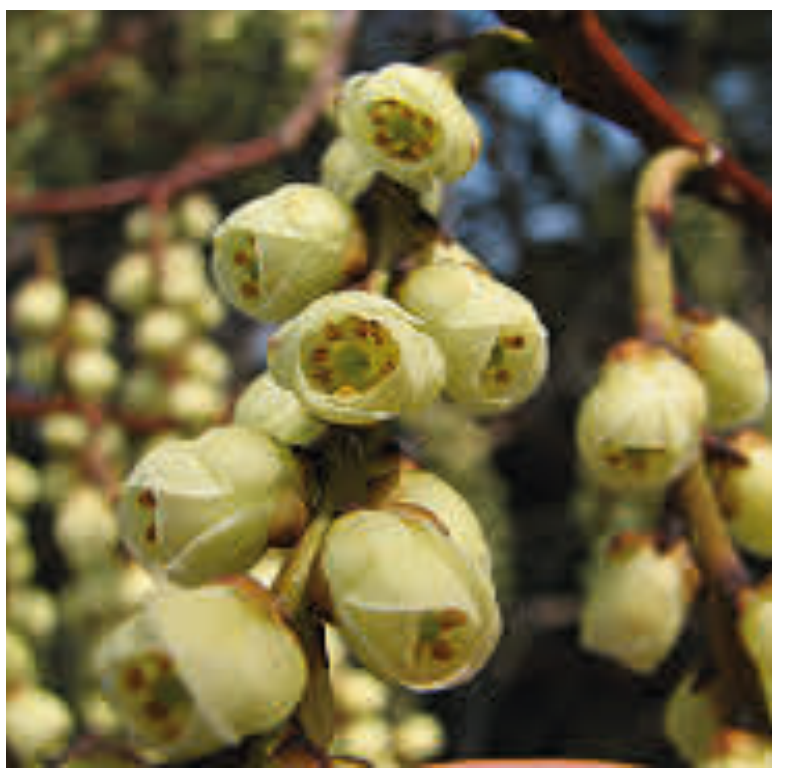

Es empfiehlt sich, die Sträucher vor einem dunklen Hintergrund wie z. B. vor immergrünen Koniferen zu pflanzen. Hier kommen die hängenden Blütenstände besonders gut zur Geltung. Der Zierwert der grünen, sonnenseits rötlich überlaufenen Beerenfrüchte ist zu vernachlässigen. Möglicherweise könnte dieser grazile, wenn auch etwas anspruchsvolle Strauch eine attraktive Alternative zu den häufig gepflanzten Forsythien darstellen und vermehrt in Gärten gepflanzt werden.

Perlschweif ist auch im Palmengarten vorhanden. Stachyurus praecox 'Rubrifolius' gedeiht im Steingarten und zeichnet sich durch eine leuchtend rote Herbstfärbung aus.

\section{Literatur}

KRÜssmann, G. I978: Handbuch der Laubgehölze. - Berlin, Hamburg.

Mabberley, D. J. 2008: Mabberley's Plant Book. 3. Aufl. - Cambridge.

Roloff, A. \& Bärtels, A. 1996: Gehölze. - Stuttgart.

Abb. 7 (oben links): Perlschweif mit Herbstfärbung. Abb. 6 (oben rechts): Detail des Blütenstandes.

Abb. 8 (unten): Bereits im Herbst angelegte Blütenknospen für das Folgejahr. 